\title{
Cavanagh and Leclerc shape-from-shadow pictures: Do line versions fail because of the polarity of the regions or the contour?
}

\author{
John M Kennedy, Juan Bai \\ Department of Psychology, University of Toronto, 1265 Military Trail, Scarborough, Ontario M1C \\ 1A4, Canada; e-mail: kennedy@scar.utoronto.ca \\ Received 2 August 1999, in revised form 4 November 1999
}

\begin{abstract}
Shape-from-shadow perception fails when the contour bordering a shadowed area is reduced to a black line, and the shadow area becomes white. It might be that the polarity of the shadowed and illuminated areas has to be from dark on the shadowed side to light on the illuminated side for successful perception. Or it may be that the line, which has two contours, has one too many for shape-from-shadow processing. Alternatively, the problem might be that one of the contours of the line is incorrectly polarised. To test these explanations, three shapefrom-shadow figures were prepared, each depicting the same referent-an elderly person. All three figures had two correctly polarised areas. One figure had a correctly polarised contour at the border between the areas. One had two correctly polarised contours. The other had one correctly polarised contour and one incorrectly polarised contour. The referent of the figure with one incorrectly polarised contour was the one difficult to make out. The result has implications for several theories, including an account of a demonstration by Hering involving penumbra.
\end{abstract}

\section{Introduction}

Kennedy (1997) demonstrated an interesting effect when the contour of a black-and-white shape-from-shadow picture is changed to a black line, and both of the areas neighbouring the line are white. He reported perception of the referent of the picture is lost. We test here three possible explanations of this effect: one to do with the polarity of the shadowed and illuminated areas, another based on the polarity of the contour between the areas, and still another drawing attention to the number of the contours between the areas.

A black-and-white picture, in which the black part represents a shadowed area and the white part represents an illuminated area, is shown in figure 1a (adapted from Mooney 1957, page 220). The referent of the picture (what is depicted by the image) is an old person. This kind of picture is called a shape-from-shadow picture by Cavanagh and Leclerc (1989). It is a bi-quantised picture (Liu and Chaudhuri 1997) in that it is divided into a dark area and a light area, with no intermediate shade of gray. There is an abrupt contour at the division between the dark and the light regions. The light region depicts the illuminated part of a referent object, and the dark region includes the part in shadow. We can call the luminance contour of the dark region a 'border' or 'coastline' of a graphic image, in the sense that it is a division between a dark region and a light region in a stimulus display. The division in the graphic image does not stand for a boundary of the surface of the referent (the old person). That is, it does not correspond to an abrupt change in depth (a figure-ground edge), as would occur at the occluding bounds of a profile of a face. Instead, it stands for the border of a shadow on the continuous surface of the referent.

Figure $1 \mathrm{~b}$ is another version of figure la (see Kennedy 1993, page 42). It was created by reducing the regions in figure la to a black line. In this black-line version of figure 1a the line has one contour that corresponds to a shadow border, and in addition it has a second contour alongside, closely paralleling the shadow-border contour. As a result, it has the shape of the border of a shadow on the surface of the referent. Hence, the line does not correspond to occluding boundaries of surfaces of a referent, as would be true for a line in an outline drawing. 


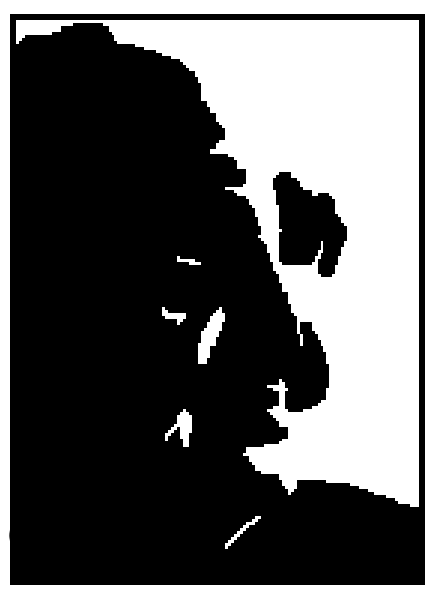

(b)

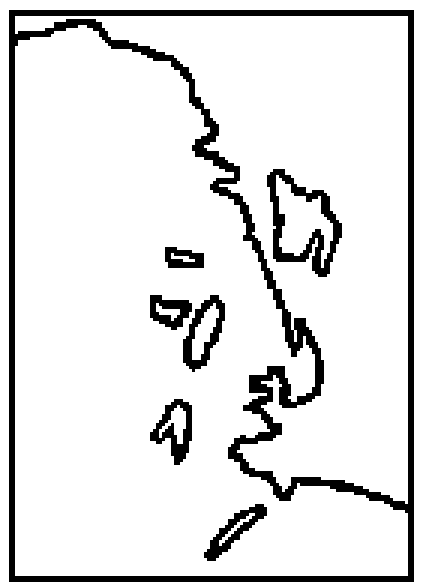

Figure 1. (a) A shape-from-shadow picture from Mooney (1957), and (b) its line counterpart. The referent is an elderly person, shown in three-quarter view, facing to the right. The central border runs from the referent's brow, via the nose, mouth, and chin to the shoulder. The upper right patch depicts one eye of the referent. If necessary, holding the picture afar, so it subtends a small visual angle, can help make the referent evident.

Although the line in figure $1 \mathrm{~b}$ is congruent with the shape of the contour in figure 1a, the perceptual results are quite different. So far as vision is concerned, only the image in figure 1a clearly resembles a face of an old person.

Why does the line version fail to evoke shape-from-shadow perception? To begin to answer this question, first let us define some terms. Shape-from-shadow displays use shadow patterns to support perception of an object in directional illumination. Some authors call these 'shape-from-shading displays' (Ramachandran 1988; Humphrey et al 1996; Mallot 1997). The term 'shape-from-shadow' will be used here because the term 'shading' is ambiguous. In the computational literature, it refers to variation due to attached shadows. Variation in shading can also be defined as "the variation of reflected flux with the angle between the incident light and the surface normal" (Cavanagh and Leclerc 1989, page 3; see also Ikeuchi and Horn 1981; Woodham 1981, 1984; Pentland 1982). This kind of shading variation "can give information concerning surface orientation in areas receiving direct illumination" (Cavanagh and Leclerc 1989, page 3). Shading need not involve a shadowed region, where illumination fails to reach a region of surface. Further, shading could be a local feature of the reflectance of the surface itself, as in the shades of a hue of a leaf. In this case shading has nothing to do with shadow. Rather, a shadow has to do with reduced illumination. "A shadow area is blocked from direct illumination" (Cavanagh and Leclerc 1989, page 3; see also Beck 1972; Yonas 1979; Berbaum et al 1983; Gilchrist et al 1983; Shafer 1985; Kennedy et al 1995).

Cavanagh and Leclerc (1989) argued that, for stimuli like figure 1a, "the only requirements for the perception of depth due to shadows" were that (i) "shadow regions be darker than the surrounding, nonshadow regions" and (ii) that "there be consistent contrast polarity along the shadow border" (page 3). There are two factors in this claim, one being the polarity of the two regions, and the other the polarity of the abrupt contour that borders these two regions. The term 'polarity' can refer to the direction of change of luminance between two extended regions. It can also refer to the change of luminance along the coastline dividing them. In both cases, the polarity is from dark on the shadowed side to light on the illuminated side. If the polarity was reversed, the result would be a negative, with the illuminated region represented by a patch of the image with darker reflectance than the shadowed region. 
It is well known that the referents of negatives are harder to see than the referents of positives (Kennedy 1993). This difficulty with negatives could be due to several factors. First, it could be due to the presence of inappropriate, 'reverse' polarity between the regions, or, second, to the inappropriate polarity of the contour. In addition, thirdly, it could be that in a negative the correct polarity of the regions is absent, a hypothesis subtly different from the first, which requires the actual presence of reverse polarity.

Interestingly, in figure $1 \mathrm{~b}$ the polarity of the regions in the image is not reversed. Rather, they are equal in reflectance-both white. Evidently, it may not just be the presence of a reversal that matters, but rather the absence of the correct black-white polarity. Alternatively, does the contour play a role? If the regions are correctly polarised, does the contour's polarity influence shape-from-shadow perception?

Hayes (1988) described shadow regions as relatively low-spatial-frequency phenomena compared to contours, and contours in these terms are high-spatial-frequency phenomena. Our purpose, in these terms, is to assess the consequences for perception of varying the polarisation of the high-frequency borders of shape-from-shadow displays.

Figure la has two regions, one black and one white. It has one contour. Figure $1 \mathrm{~b}$ has two white regions and a thin black line between them. The black line has two contours. Two physical factors - the polarity of regions and the polarity of contoursmay be relevant to figure 1a. Also, at least three factors may be relevant to the effects in figure $1 \mathrm{~b}$ : the polarity of the regions, the polarity of the contours, and the number of the contours.

Consider three explanations of the perceptual effects of figure $1 \mathrm{~b}$.

(i) The failure in shape-from-shadow perception of figure $1 \mathrm{~b}$ could be due to the lack of the correct polarity between the two large regions: Both the region that should be in shadow and the illuminated region are depicted by white areas.

(ii) The failure of figure $1 \mathrm{~b}$ could be due to the presence of an incorrectly polarised contour. Physically, one of the two contours of the line is incorrectly polarised. The line has one contour with the region that should be in shadow and a second contour with the region that should be illuminated. The contour bordering the area that should be in shadow has white on one side- on the side of the area that should be shadow. It has black on the other side-the blackness of the line itself. The contour bordering the region that is illuminated has white on the side of the illuminated region and the blackness of the line on the other side. The two contours of the line are polarised in opposite directions. The contour bordering the illuminated region is correctly polarised physically, since the illuminated region is white, and the opposite side of that contour is black. The contour bordering the region that should be in shadow is incorrectly polarised physically, since the region that should be in shadow, and relatively dark, is shown in white, and the blackness of the line is on the opposite side of the contour. It is polarised like a photographic negative. It is light where it should be dark, and black where it should be light. This incorrectly polarised contour may prevent shapefrom-shadow perception from being effective, even though there is a second contour adjacent to it with the correct polarity.

(iii) The failure of figure $1 \mathrm{~b}$ may be due to the presence of two contours in figure $1 \mathrm{~b}$, in place of the single contour in figure 1a. That is, the line in figure $1 \mathrm{~b}$ has two contours, and shape-from-shadow perception may fail if more than one contour is perceptually evident. The number of contours may inconvenience shape-from-shadow perception quite independently of the polarity of the contours.

To test these three explanations, three pictures (figures $2 a, 2 b$, and $2 c$ ) were created from figure la.

The first picture was formed by adding gray to the line version of figure 1 in the area that should be in shadow (or, equivalently, reducing the black shadow region in figure la to gray, and adding a black line to the border of the gray shadow). The result 


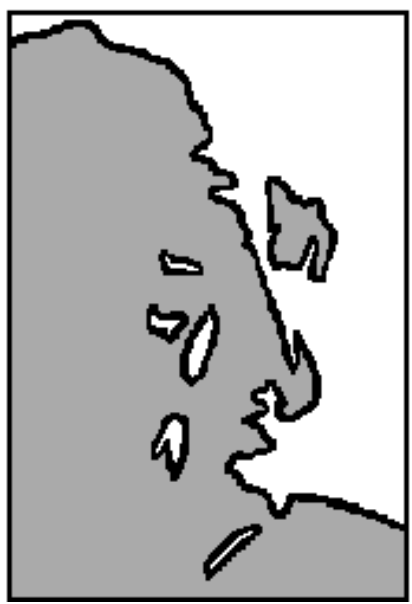

(a)

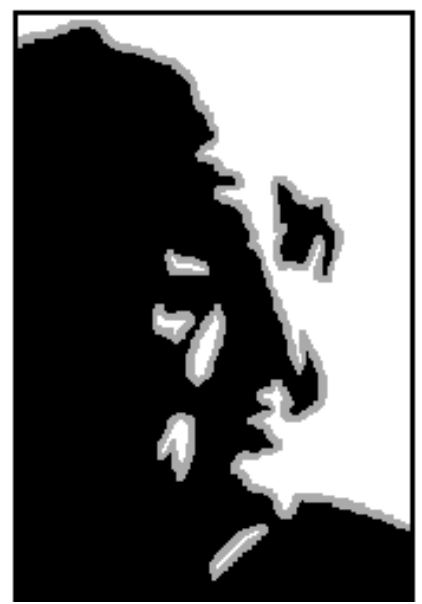

(b)

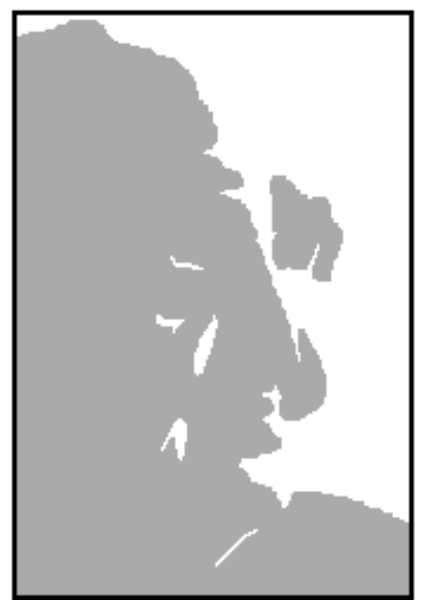

(c)

Figure 2. Three figures created from figure 1a: (a) the 'black line' version, (b) the 'gray line' version, and (c) the 'gray control' version.

is shown in figure $2 \mathrm{a}$. For the sake of convenience, figure $2 \mathrm{a}$ can be called the 'black line' version. As in figure $1 \mathrm{a}$, the polarity of the large regions in figure $2 \mathrm{a}$ is from dark in the region that should be in shadow to light in the region that should be illuminated. Also, as in figure 1b, the border between the two large areas in figure $2 \mathrm{a}$ has two contours. One contour has the same polarity as the division in figure 1a (dark on the shadowed side and light on the illuminated side). The other contour, running alongside it, has the reverse polarity (darker on the illuminated side).

If successful perception of the referent depends chiefly on the polarity of the large regions in the image [explanation (i)], then figure $2 \mathrm{a}$ should behave like figure $1 \mathrm{a}$. It should succeed in shape-from-shadow perception, since the two large regions have the same polarity. Also, if it is largely the presence of a contour with the correct polarity that matters and a second contour is immaterial it should succeed in shape-fromshadow perception, since it has the same contour as in figure la.

Alternatively, the presence of a contour with incorrect polarity alongside the shadow region may prevent shape-from-shadow perception [explanation (ii)]. That is, despite the presence of a correctly polarised contour, difficulties may be posed by the presence of the 'negative' contour, and shape-from-shadow perception of figure $2 \mathrm{a}$ may fail.

Further, the account based on the presence of two contours [explanation (iii)] contends shape-from-shadow perception of figure 2 a should fail. It has one contour too many, the theory notes.

In figure $2 \mathrm{~b}$, the shadowed region is black and the illuminated region is white, but a gray line is provided on the border between these two regions. As a result, the polarity of the large regions is from dark in the shadowed area (black) to light in the illuminated area (white). The polarities of both of the two luminance contours of the gray line are also from dark on the shadowed side to lighter on the illuminated side (from black to gray and gray to white). We can call this the 'gray line' version.

Both of the two hypotheses about polarity-explanation (i) about regions and explanation (ii) about contours - predict successful shape-from-shadow perception of the old person in figure $2 \mathrm{~b}$. However, explanation (iii) contends that the presence of more than one contour at the division between the large regions can prevent shapefrom-shadow processing.

Figure $2 \mathrm{c}$ is gray in the shadowed region and light in the illuminated region. It can be used to ensure that the basic pattern modified in figures $2 \mathrm{a}$ and $2 \mathrm{~b}$ can be seen by 
subjects as depicting the referent. It uses a gray shadow region like figure $2 \mathrm{a}$. It is like figure 1a in both the polarity of the regions and the polarity of the coastline. It satisfies the Cavanagh and Leclerc (1989) requirements for successful shape-from-shadow perception.

Figure 2c can be called the 'gray control' version. All three hypotheses predict figure $2 \mathrm{c}$ should succeed, but figure $2 \mathrm{c}$ provides a baseline against which to assess the others.

\section{Method}

\subsection{Participants}

Nine university students (seven women and two men; mean age $=21.6$ years, $\mathrm{SD}=0.7$ years) participated in the experiment as a class demonstration, which they selected from a series of demonstrations. All had normal or adjusted to normal vision.

\subsection{Materials}

The images in figure 2 were shown on a Dell Latitude LM notebook computer with a Pentium $133 \mathrm{MHz}$ microprocessor and an SVGA active-matrix display, $30 \mathrm{~cm}$ on the diagonal. The screen of the notebook was vertical and at about the same level as the subject's eyes. The distance from the subject's eyes to the screen of the notebook was about $70 \mathrm{~cm}$. The rectangular frame of each image on the display measured $9.5 \mathrm{~cm}$ by $7.3 \mathrm{~cm}$.

\subsection{Procedure}

All three figures in figure 2 were shown to each subject, one at a time. The first six subjects each used one of the six possible orders of the three figures. The remaining three subjects used three of the six orders, a different order per subject. Each subject conducted the experiment individually.

Two demonstration pictures were shown to the subjects, one at a time. They were a shape-from-shadow picture of a man's face and its line counterpart (from Kennedy 1997, page 65). The subjects were told that the referent of the picture was a man. Then, each subject was told that people might rate the shape-from-shadow picture as 8 on a 10-point scale (10 being very easy) in terms of how easy it is to see the person in the picture. They were also told the line version might be rated as 2 on the same scale, meaning it is difficult to see the person in this version. Numbers less extreme than 1 and 10 were chosen for the example so that subjects would have room on either side of the example numbers for their responses. Next, the black-and-white shapefrom-shadow picture in figure la was shown to the subject, and the referent in the picture - an old person - was mentioned to the subject. The eye, the tip of the nose, the mouth, and the shoulder of the referent were pointed out to the subjects. All of the subjects reported being able to see the old person. Next, the subjects were told they were going to see some other versions of the same picture. Then, the three pictures in figure 2 were shown one at a time. The subjects were asked to rate how easy it was to see the old person in each of the three figures, using the 10-point scale. All subjects responded within a few seconds.

\section{Results}

The mean rating on the ease-of-perception scale for figure 2c (the 'gray control' version) was $7.11(\mathrm{SD}=1.27)$, for figure $2 \mathrm{~b}$ (the 'gray line' version) was $6.44(\mathrm{SD}=0.88)$, and for figure 2a (the 'black-line' version) was $2.67(\mathrm{SD}=1.32)$ (one-way repeated-measures ANOVA, $\left.F_{2,16}=54.21, p<0.0001\right)$. An a posteriori $t$-test found the 'black-line' version to be rated significantly lower than the 'gray control' version $\left(t_{8}=6.904, p<0.001\right)$ and the 'gray line' version $\left(t_{8}=11.795, p<0.001\right)$. There was no significant difference between the 'gray control' and the 'gray line' version $\left(t_{8}=1.512, p>0.05\right)$. All $p$ values have been Bonferroni adjusted. 
Evidently, the presence of the wrongly polarised 'negative' contour diminishes the ease of perception of the shape-from-shadow figures.

\section{Discussion}

The 'gray line' version allowed shape-from-shadow perception about as readily as the 'gray control' version. The 'black-line' version was inferior to both. The results support the view that as long as the polarities of the contours at the coastline proceed from dark to light in the direction from shadow to illuminated regions, shape-from-shadow perception remains successful. We have tested pictures with additional referents and the effects are the same as those found with figure 2.

In sum, the results support an explanation of effects of figure $1 \mathrm{~b}$ emphasising the polarity of the contour, and run counter to the accounts stressing the polarity of its large regions or the number of the contours.

What happens when the wrongly polarised 'negative' contour impedes perception? Figure $1 \mathrm{~b}$ and figure $2 \mathrm{a}$ often look flat like maps or like flat patches with a background. By patches we mean flat surfaces with borders, in front of the background, ie figure-ground edges (Rubin 1915; Kennedy 1974). Nakayama et al (1989) call these 'intrinsic' edges. The figure-ground percept is a result, not a cause, we suggest. Peterson (1999, page 276) notes "figure-ground segregation is one possible organization that can be perceived when two adjacent regions share a contour". As Peterson notes, in a valuable discussion of several perceptual options for borders, figure-ground is one of several options for a border (see also Tse 1998). Figure-ground involves an edge of a surface a step in depth but a shadow lies on a continuous surface. At a shadow border, the figure or frontal surface runs through the border and the figure bears the shadow. The shadow lies on the figure or frontal surface. When shape-from-shadow fails, the contour can be organised as an edge with depth, the figure-ground option.

There are several aspects of shape-from-shadow perception that deserve attention in light of our results. In shape-from-shadow perception, a relatively dark area is perceived as a shadow, and a relatively light area is perceived as illuminated. And, in addition, the shadowed and illuminated areas are perceived as related to the shape of a surface (say, a face) on which the shadow and illumination lie. Since there are two factors here, it is conceivable that on occasion the surface shape (the face) might be evident, but the dark and light areas might be misinterpreted as stained areas, not shadows (eg as 'port-wine stains' like Mr Gorbachev's forehead skin-spot). This might be called a failure to identify an area as a shadow, or failure of 'shadow perception' while shapefrom-shadow succeeds. Further, it is conceivable that an area could be perceived as being in shadow, while the shape of the surface bearing the shadow is misperceived. (We might report we see an unfamiliar object bearing shadows, and fail to see the face.)

Observation suggests that in our figures, when the presence of a line prevents shape-from-shadow perception of the referent, the dark area is not seen as a shadow on a surface moulded in three dimensions like a face. Rather, it is seen as a flat black stained region (which of course it is) like a map. Correspondingly, the contour appears very much like a coastline on a flat map. If so, this is a case in which both shadow perception and shape-from-shadow perception fail.

The distinction between perception of an area as in shadow, as opposed to the perception of the shape of the area bearing the shadow, allows interesting questions about causality.

Does identification of the dark area as a shadow fail because shape-from-shadow fails? Or is it the other way around? Or, is it a combination, both failing simultaneously? Of course, the simultaneous case would occur if shadow perception (identification of an area as a shadow) and shape-from-shadow perception (perception of the shape of the surface bearing the shadow) were not separable percepts. 
To understand what is the case in the current experiment, it might be helpful to recall an early study that also added lines at the contour of a shadow, namely Hering's famous demonstration (1874/1964; see Goldstein 1984) where a line was added to obscure a penumbra.

A penumbra is a fuzzy border, found on many shadows (see Goldstein 1984, pages $252-254)$. The penumbra is caused by illumination from an extended source of light. A point source of light casts shadows with sharp borders. An extended source results in partial shadows, with some regions of shadow receiving illumination from a fraction of the source. Regions closer to the interior of the shadow receive illumination from less of the source. Therefore, there is an illumination gradient at the border of the shadow.

The gray line of figure $2 \mathrm{~b}$ (the 'gray line' version) has properties in common with a penumbra. A penumbra usually has many intermediate shades of gray between shadow and non-shadow. The gray line in figure $2 b$ is an intermediate shade of gray between the black shadow and the white non-shadow. However, it is not graded, and it has sharp, not fuzzy, contours.

In his famous 'ringed shadow' experiment, Hering (1874/1964) drew a black line along the contour of a cast shadow. His line covered the penumbra (as shown in Goldstein 1984, his figure 9.28). The shadowed area often does not appear to be a shadow. It often looks like a dark spot or a stain.

One might attribute the failure of shadow perception in Hering's demonstration to the absence of a penumbra. But it is evident from figures 1a and 2c that sharpcontoured figures can support shadow perception and shape-from-shadow perception. A more likely explanation is that, because of the added black line, there is a contour polarised from gray on the shadowed side to black in the direction of the nonshadowed side, physically the inverse polarity of a shadow border. Accordingly, perhaps the reason for the failure of shadow perception in Hering's demonstration is that one of the contours of the line offers inappropriate polarity, the same account we can give for the failure of figure $2 \mathrm{a}$.

Hering's demonstration is usually discussed in terms of perception of an area as a shadow. That is, it is related to shadow perception rather than shape-from-shadow perception. Likely, however, shape-from-shadow perception is relevant. The observer in Hering's case can see that shadow falls on a flat surface-a wall. When the dark area is perceived as a stain, it looks like a stain on a flat wall. [In Goldstein's (1984) illustration, the shadow is cast on a flat horizontal surface-a floor.] The surface shape is seen, in addition to the dark region appearing to be a stain. We might say 'shape from stain' perception occurs. But there is a confound. The surface is seen as flat when seen as bearing a stain, and also flat when seen as having a shadow cast on it.

Since the surface bearing the shadow is flat in both cases, the Hering demonstration does not distinguish shape-from-shadow and the shape perceived from the stain. In figure 2, however, when shape-from-shadow perception succeeds, the surface bearing the shadow is perceived as being three-dimensional, but when a line makes shapefrom-shadow fail, the shape of the surface bearing the shadow is perceived as flat and resembling a map. Therefore, not only is the shadow interpreted as a dark region or stain due to the added line, the shape of the perceived surface changes from a face in three dimensions to a flat pattern. So we may say that a contour with a polarity unsuitable for a shadow destroys two aspects of perception: shape-from-shadow and perception of a shadow.

We should point out that the present experiment shows that a sharp-contoured display with black, white, and an intermediate shade of gray, such as the 'gray line' version in figure $2 \mathrm{~b}$, induces shape-from-shadow perception. Obviously, having only two levels of luminance, and one salient change in luminance, is not a necessary requirement for successful shape-from-shadow perception in displays without graded 
borders. The display can have three discrete levels of luminance. Presumably, it can have many discrete levels and can still succeed. And, of course, a graded display, such as one with a penumbra, has unlimited numbers of gray levels and still succeeds in shape-from-shadow perception and shadow perception.

Our results raise interesting questions about contours, lines, and luminance, especially since there are many kinds of contours besides luminance borders. For example, it would be worth testing whether the effects of physically incorrect polarisation might be mimicked by a line with an illusory border that had apparent incorrect polarisation. Also, similar effects might arise from lines with some binocular or motion-defined borders. Further, if the darkness of the shadow area is provided by gratings, the termini of the grating lines at the border can be varied in many ways to explore luminance variables, such as the need for consistent polarity, as mentioned by Cavanagh and Leclerc (1989). How much of the border needs consistent polarity is an open question, but one vital to shape-from-shadow. Certainly, shape-from-shadow requires an extended border, and an overall form, since a short stretch of contour could be a stain or a surface edge rather than a shadow. In addition, the border in a digitised shape-fromshadow image can be moved by varying the digitisation threshold. This variable may be especially relevant to penumbra and attached shadows on curved surfaces. Also, the thickness of lines that can block shape-from-shadow analysis deserves investigation.

In summary, our results indicate a wrongly polarised contour bordering a shadow region can block shape-from-shadow perception and perception of a region as a shadow. This can explain why reduction of a shape-from-shadow picture to a line results in loss of perception of the picture's referent. Explanations of this loss in terms of large regions and their polarity, or the presence of a pair of contours, are not supported.

Acknowledgements. We thank Stuart Anstis, Ian Howard, P Muter, Allison B Sekuler, I Juricevic, M D Regan, Denis Pelli, and three anonymous reviewers for comments.

\section{References}

Beck J, 1972 Surface Color Perception (Ithaca, NY: Cornell University Press)

Berbaum K, Tharp D, Mroczek K, 1983 "Depth perception of surfaces in pictures: looking for conventions of depiction in Pandora's box" Perception 12 5-20

Cavanagh P, Leclerc Y G, 1989 "Shape from shadows" Journal of Experimental Psychology: Human Perception and Performance $153-27$

Gilchrist A, Delman S, Jacobsen A, 1983 "The classification and integration of edges as critical to the perception of reflectance and illumination" Perception \& Psychophysics 33 425-436

Goldstein E B, 1984 Sensation and Perception (Belmont, CA: Wadsworth)

Hayes A, 1988 "Identification of two-tone images; some implications for high- and low-spatialfrequency processes in human vision" Perception $17429-436$

Hering 1874/1964 Outlines of a Theory of the Light Sense translated into English by L M Hurwich, D Jamieson (Cambridge, MA: Harvard University Press) [originally published in 1874]

Humphrey G K, Symons L A, Herbert A M, Goodale M A, 1996 "A neurological dissociation between shape from shading and shape from edges" Behavioural Brain Research $76117-125$

Ikeuchi K, Horn B K P, 1981 "Numerical shape from shading and occluding boundaries" Artificial Intelligence $15141-184$

Kennedy J M, 1974 A Psychology of Picture Perception (San Francisco, CA: Jossey-Bass)

Kennedy J M, 1993 Drawing and the Blind (New Haven, CT: Yale University Press)

Kennedy J M, 1997 "How the blind draw" Scientific American 276 (January) 60-65

Kennedy J M, Nicholls A, Desrochers M, 1995 "From line to outline", in Drawing and Looking Eds C Lange-Küttner, G V Thomas (London: Harvester Wheatsheaf) pp 62-74

Liu C H, Chaudhuri A, 1997 "Learning faces through bi-quantised pictures", in Studies in Perception and Action IV Eds M A Schmuckler, J M Kennedy (Mahwah, NJ: Lawrence Erlbaum Associates)

Mallot H A, 1997 "Spatial scale in stereo and shape from shading: image input, mechanisms, and tasks" Perception $261137-1146$

Mooney C M, 1957 "Age in the development of closure ability in children" Canadian Journal of Psychology $11219-226$

Nakayama K, Shimojo S, Silverman G H, 1989 "Stereoscopic depth: its relation to image segmentation, grouping, and the recognition of occluded objects" Perception $1855-68$ 
Pentland A P, 1982 "Finding the illuminant direction" Journal of the Optical Society of America 72 $448-455$

Peterson M A, 1999 "What's in a stage name? Comment on Vecera and O'Reilly (1998)" Journal of Experimental Psychology: Human Perception and Performance 25 276-286

Ramachandran V S, 1988 "Perceiving shape from shading" Scientific American 259 (August) 76-83

Rubin E, 1915/1958 Synsoplevede Figurer (Copenhagen: Gyldendalske Boghandel) [translated into English as "Figure and ground", in Readings in Perception Eds D C Beardslee, M Wertheimer (1958, Princeton, NJ: Van Nostrand) pp 194-203]

Shafer S A, 1985 Shadows and Silhouettes in Computer Vision (Boston, MA: Kluwer Academic)

Tse P U, 1998 "Illusory volumes from conformation" Perception 27 977-992

Woodham R J, 1981 "Analyzing images of curved surfaces" Artificial Intelligence 17 117- 140

Woodham R J, 1984 "Photometric method for determining shape from shading", in Image Understanding 1984, Eds S Ullman, W Richards (Norwood, NJ: Ablex Publishing) pp 97-125

Yonas A, 1979 "Attached and cast shadows", in Perception and Pictorial Representation Eds C F Nodine, D F Fisher (New York: Praeger) pp 100-109 